\title{
Graded TiN Coating by Supersonic Free-Jet PVD Combined with Reactive Plasma
}

\author{
Atsushi Yumoto $^{1, *}$, Takahisa Yamamoto ${ }^{2}$, Fujio Hiroki $^{1}$, Ichiro Shiota ${ }^{3}$ and Naotake Niwa ${ }^{1}$ \\ ${ }^{1}$ Department of Mechanical Systems Engineering, Faculty of Engineering, Kogakuin University, Tokyo 163-8677, Japan \\ ${ }^{2}$ Department of Advanced Materials Science, Graduate School of Frontier Sciences, University of Tokyo, Tokyo 113-8656, Japan \\ ${ }^{3}$ Department of Materials Science and Technology, Faculty of Engineering, Kogakuin University, Tokyo 192-0015, Japan
}

\begin{abstract}
Ceramic coating is a very popular technology for improving the properties of structural materials. A titanium nitride (TiN) coating is a typical example and has been widely applied to cutting tools, electronic devices and many other fields utilizing its superior physical properties. This paper sought to produce a graded TiN coating on a Ti substrate by combining Supersonic Free-Jet PVD (SFJ-PVD) with a reactive plasma-metal reaction technique. The authors have developed SFJ-PVD as a new coating method in which a coating film is formed by depositing nanoparticles with very high velocity onto a substrate. SFJ-PVD can provide a high deposition rate and thick film coating. Gradually changing the nitrogen flow rate during deposition produces a graded TiN coating, in which composition changes gradually from pure Ti to TiN. A monolithic TiN coating is also produced with SFJ-PVD. XRD analysis of the graded TiN detected peaks for Ti, $\mathrm{Ti}_{2} \mathrm{~N}$ and TiN, while only a TiN peak is observed in the monolithic TiN coating. EPMA analysis of a graded coating reveals a gradual compositional change from pure Ti to TiN. Few pores or cracks are observed in a graded TiN or in a monolithic TiN formed under the optimized conditions of SFJ-PVD.
\end{abstract}

(Received July 29, 2002; Accepted September 30, 2002)

Keywords: physical vapor deposition, supersonic gas flow, nanoparticle, functionally graded material, titanium nitride

\section{Introduction}

Ceramic coating is a very popular technology for improving the properties of structural materials. A titanium nitride (TiN) coating is a typical example and has been widely applied to cutting tools, electronic devices and other industrial applications utilizing its high hardness at elevated temperature, high melting point, high thermal conduction, and other superior properties.

Various methods such as ion plating, ${ }^{1-4)}$ sputtering $^{5-7)}$ and $\mathrm{CVD}^{8,9)}$ have been used to form TiN coatings. These methods have shortcomings, such as low deposition rate and difficulty in obtaining a thick, high-density film. Cracks and many pores are often observed in the coated film. Moreover, it is difficult to obtain sufficient bonding strength at an interface by direct coating of nitrides on a substrate metal because of residual stress caused mainly by the difference of thermal expansion coefficients between ceramics and substrate metals. ${ }^{10)}$ Recently, compositional grading from the interface in a metal/ceramic composite coating has received attention because it reduces the residual stress. This concept was successfully applied to prepare metal/ceramic composite coatings. ${ }^{10-15)}$

The authors developed a new coating method called "supersonic free-jet PVD (SFJ-PVD)". ${ }^{16-20)}$ In SFJ-PVD, a coating film is formed by depositing nanoparticles at very high velocity onto a substrate. The schematic diagram of SFJ-PVD is illustrated in Fig. 1, showing the two stages in SFJ-PVD, "gas evaporation" and "vacuum deposition". In the gas evaporation stage, a source material is evaporated to form nanoparticles in an inert gas atmosphere. The nanoparticles are then carried to a substrate with the inert gas through a transfer pipe where a gas flow is generated by the pressure difference between the evaporation and deposition chambers. The gas flow is accelerated to supersonic velocity (Mach 3.6) by a specially

*Graduate Student, Kogakuin University.

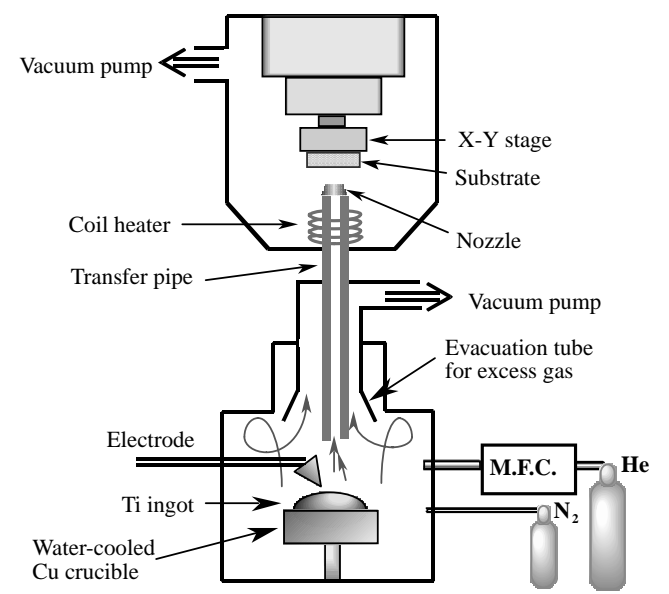

Fig. 1 Schematic diagram of supersonic free-jet PVD apparatus.

designed supersonic nozzle joined to the tip of the transfer pipe. In the deposition stage, the nanoparticles are deposited onto the substrate in the deposition chamber.

The authors succeeded in obtaining a uniform, highdensity, thick metallic coating of $\mathrm{Al}$ or Ti on metal substrates with SFJ-PVD. ${ }^{16,19)}$ M. Uda, S. Ohno et al. ${ }^{21-23)}$ and other researchers ${ }^{24)}$ produced nitride particles with a reactive plasmametal reaction technique. They produced the nitride particles with a gas evaporation process in an $\mathrm{N}_{2}$ atmosphere.

This paper sought to produce a graded TiN coating on a Ti substrate by combining SFJ-PVD with the reactive plasmametal reaction technique.

\section{Experimental Procedure}

The purity of the $\mathrm{Ti}$ source for evaporation exceeded 99.999 mass $\%$. The source material was placed in a watercooled copper crucible then heated and evaporated by arc plasma. The $20 \mathrm{~mm} \times 20 \mathrm{~mm}$ substrate was fixed on an $\mathrm{X}$ $\mathrm{Y}$ stage that was driven in $\mathrm{X}-\mathrm{Y}$ directions, and the coated area 
was $5 \mathrm{~mm}$ square. The stage and the nozzle are heated by electric resistance heating systems.

The evaporation chamber is equipped with an evacuation tube with a needle valve to evacuate excess gas that is not suctioned through the transfer pipe. The evacuation tube also suppresses gas stagnancy caused by the excess gas, which in turn reduces coarse particles by suppressing the stagnancy and secondary agglomeration of particles.

To form a graded TiN coating, we began evaporating Ti in a purified He gas atmosphere and gradually increased the flow rate of $\mathrm{N}_{2}$ gas. The preparation conditions are shown in Table 1.

We performed SEM and TEM observations and EPMA analysis on the cross section of coating films. We also conducted X-ray diffraction (XRD) analysis of the coated area and compared it with the XRD profile of the substrate because in our samples, the XRD profile of coated samples might contain the profile of a $5 \mathrm{~mm}$ square coated area as well as the profile of the substrate area surrounding it.

We produced a monolithic TiN coating on an SUS304 substrate to confirm the possibility of producing a TiN singlephase film with SFJ-PVD by controlling the flow rate of $\mathrm{N}_{2}$ gas because a graded TiN coating film might contain TiN as well as other phases. The SUS304 substrate was chosen to eliminate the XRD profile of Ti.

\section{Results and Discussion}

Figure 2 shows the surface of a graded coating from Ti to TiN on a Ti substrate. The photograph shows that there were no cracks in the coating. The XRD profiles of a graded

Table 1 Preparation conditions of TiN or graded TiN coatings.

\begin{tabular}{lcc} 
& TiN coatings & graded TiN coatings \\
\hline Source material & \multicolumn{2}{c}{ Pure Ti (>99.999) } \\
Arc current (A) & \multicolumn{2}{c}{20} \\
Nozzle heater $(\mathrm{W})$ & \multicolumn{2}{c}{750} \\
Substrate temperature (K) & \multicolumn{3}{c}{773} \\
\hline Substrate materials & SUS304 & \multicolumn{2}{c}{ Ti (JIS 1) } \\
He gas flow rate (L/min) & 2.0 & $3.0-2.0$ \\
$\mathrm{~N}_{2}$ gas flow rate (L/min) & 1.0 & $0-1.0$ \\
\hline
\end{tabular}

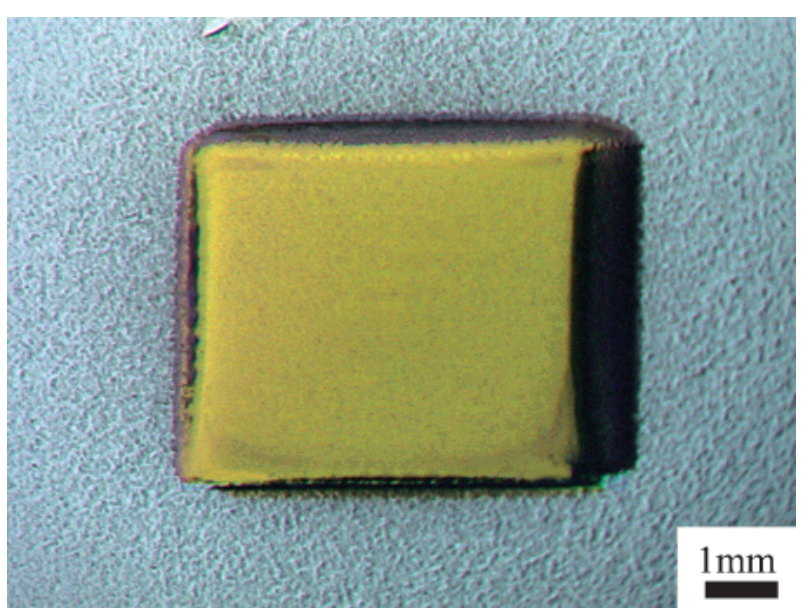

Fig. 2 Photograph of a graded TiN film surface.

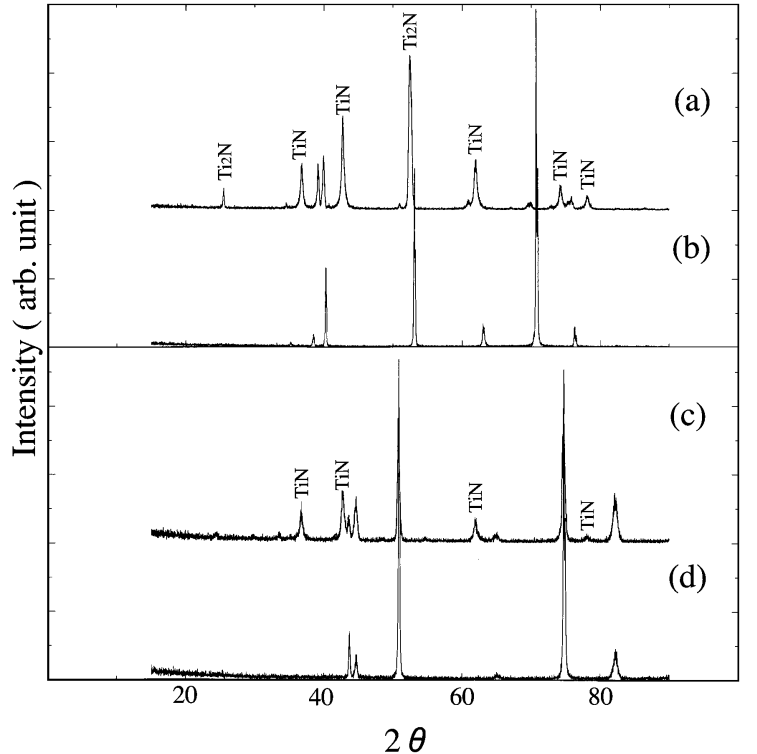

Fig. 3 X-ray diffraction profiles of (a) Graded TiN, (b) uncoated Ti substrate, (c) TiN coating on SUS304 and (d) uncoated SUS304 substrate.

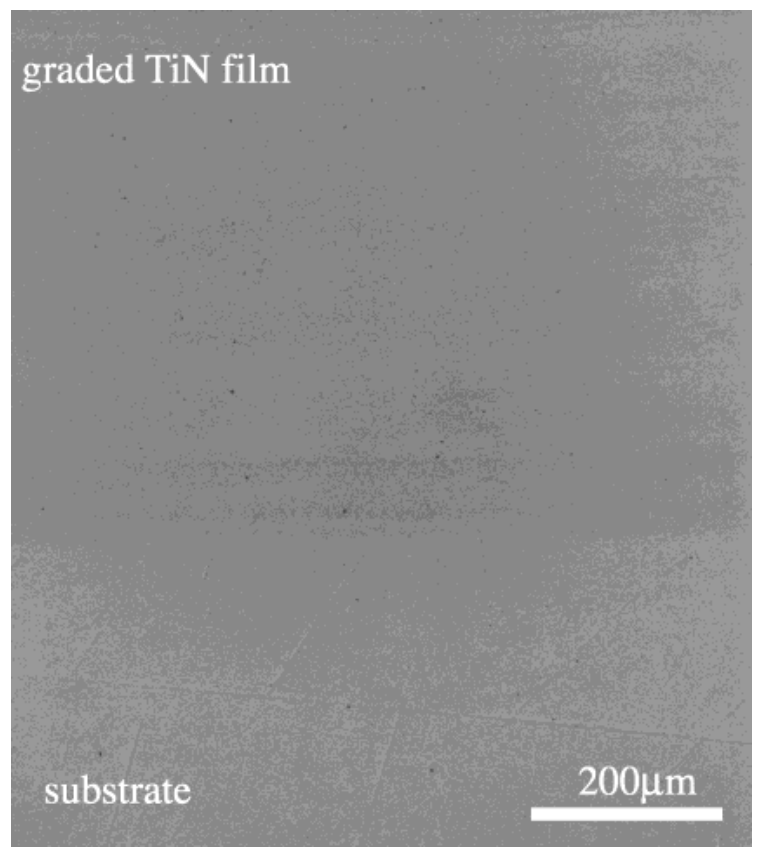

Fig. 4 Photograph of the cross-section of a graded TiN film.

TiN film on a Ti substrate, an uncoated Ti substrate, a monolithic TiN coating on an SUS304 substrate, and an uncoated SUS304 substrate are shown in Figs. 3(a), (b), (c) and (d). We measured the XRD profiles of the substrates to subtract the diffraction from the substrates around the coated areas.

The XRD profile shown in Fig. 3(a) reveals that three phases ( $\mathrm{Ti}$ metal, tetragonal sub-nitride $\mathrm{Ti}_{2} \mathrm{~N}$ and cubic $\mathrm{TiN}$ ) coexist in a graded TiN coating on a Ti substrate. The diffraction profile shown in Fig. 3(c) indicates that only TiN exists in a monolithic TiN coating on an SUS304 substrate.

Figure 4 presents a SEM photograph of the cross-section of a graded TiN coating on a Ti substrate where no pores and cracks are found.

Figure 5 shows the result of an EPMA line analysis of the cross-section of a graded TiN coating. EPMA revealed that 


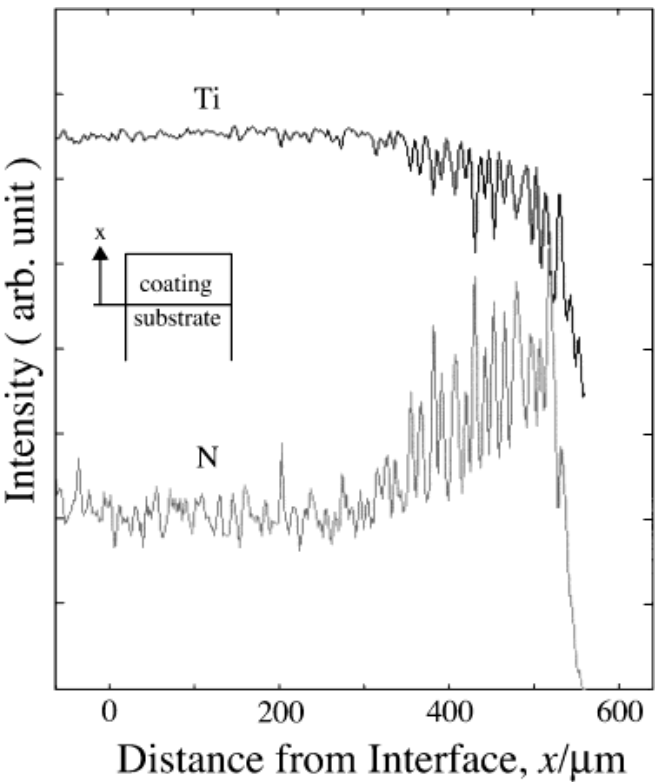

Fig. 5 EPMA line analysis of the cross-section of a graded TiN film.
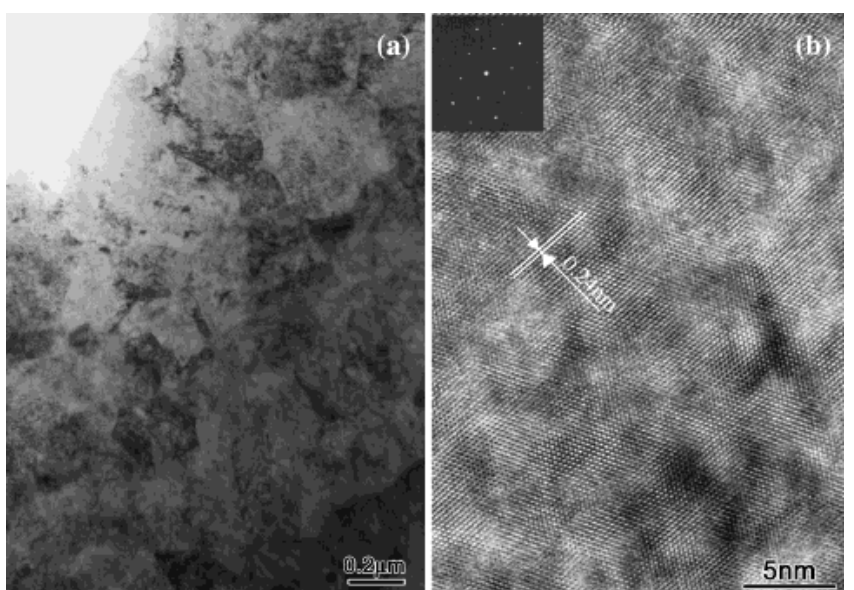

Fig. 6 TEM micrographs of the area near the surface of a graded TiN film. (a) TEM image. (b) HRTEM image and diffraction pattern of cubic TiN structure.

the coating started with pure $\mathrm{Ti}$, then gradually increased in nitrogen content as shown in Fig. 5.

Figure 6 shows TEM photographs of an area near the surface of a graded TiN coating. The TEM photographs revealed cubic TiN as detected in XRD analysis, and the grain sizes of TiN were very fine, varying from 80 to $600 \mathrm{~nm}$.

\section{Conclusions}

We successfully achieved a TiN coating and a graded TiN coating using the new SFJ-PVD technique.

The results obtained in this paper are as follows,

(1) A TiN film and a graded TiN film were produced by depositing nanoparticles formed in the evaporation chamber with a controlled atmosphere of $\mathrm{He}$ and $\mathrm{N}_{2}$.

(2) XRD analysis and TEM observation revealed the crystal structure of the TiN film to be cubic.

(3) Neither the graded TiN film nor the TiN film contains pores and cracks on the surfaces or inside the films.

(4) The grain size of $\mathrm{TiN}$ in the films is from 80 to $600 \mathrm{~nm}$.

\section{Acknowledgments}

The authors gratefully thank Mr. K. Ono and Mr. K. Fukawa of the Engineering Research Institute, School of Engineering, the University of Tokyo for the XRD analysis and Dr. M. Kosaka of the Advanced Materials Center (AMC), Kogakuin University for the EPMA analysis.

This study was supported by the Sasakawa Scientific Research Grant from The Japan Science Society, Iketani Science, AMC of Kogakuin University and Technology Foundation and Grant-in-Aid for Scientific Research (C).

\section{REFERENCES}

1) Y. M. Chem, G. P. Yu and J. H. Huang: Surf. Coat. Technol. 141 (2001) $156-163$.

2) S. Xiao, C. P. Lungu and O. Takai: Thin Solid Films 334 (1998) 173177.

3) S. Wouters, S. Kadlec, C. Quaeyhaegens and L. M. Stals: Surf. Coat. Technol. 97 (1997) 114-121.

4) Y. Inokuti, K. Suzuki and Y. Kobayashi: Mater. Trans., JIM 36 (1995) 1081-1087.

5) H. Sato, M. Sato, M. Tagami, K. Funato, M. Sasaki, S. Taguchi, S. Mizuno and M. Kobayashi: Vacuum 59 (2000) 437-444.

6) C. P. Lungu, M. Futsuhara, O. Takai, M. Braic and G. Musa: Vacuum 51 (1998) 635-640.

7) C. H. Park, Y. M. Sung and W. G. Lee: Thin Solid Films 312 (1998) $188-195$.

8) A. Larsson and S. Ruppi: Thin Solid Films 402 (2002) 203-210.

9) J. Y. Yun and S. W. Rhee: Thin Solid Films 320 (1998) 163-165.

10) X. Zeng, S. Zhang and J. Hsieh: Surf. Coat. Technol. 102 (1998) 108112.

11) B. A. Movchan: Surf. Coat. Technol. 149 (2002) 252-262.

12) I. Dahan, U. Admon, N. Frage, J. Sariel, M. P. Dariel and J. J. Moore: Surf. Coat. Technol. 137 (2001) 111-115.

13) A. Raveh, M. Weiss, M. Pinkas, D. Z. Rosen and G. Kimmel: Surf. Coat. Technol. 114 (1999) 269-277.

14) A. A. Voevodin, M. A. Capano, S. J. P. Laube, M. S. Donley and J. S. Zabinski: Thin Solid Films 298 (1997) 107-115.

15) K. Kokini, J. Dejonge, S. Rangeraj and B. Beardsley: Surf. Coat. Technol. 154 (2002) 223-231.

16) A. Yumoto, F. Hiroki, I. Shiota and N. Niwa: J. Japan Inst. Metals 65 (2001) 635-643, in Japanese.

17) A. Yumoto, F. Hiroki, I. Shiota and N. Niwa: Proc. of the Second International Conference on Light Materials for Transportation Systems (LiMAT-2001) pp. 991-996.

18) A. Yumoto, F. Hiroki, I. Shiota and N. Niwa: MASS AND CHARGE TRANSPORT IN INORGANIC MATERIALS 'Fundamentals to Devices', (P. Vincenzini and V. Buscaglia, Eds., TECHNA, 2000) pp. 991-998.

19) A. Yumoto, F. Hiroki, I. Shiota and N. Niwa: Netsu Shori 42(2002) 83-90, in Japanese.

20) A. Yumoto, F. Hiroki, I. Shiota and N. Niwa: Surf. Coat. Technol., in press.

21) M. Uda and S. Ohno: J. Surf. Sci. Soc. of Jpn. 5 (1984) 426-433, in Japanese.

22) S. Ohno, H. Okuyama, K. Honma, K, Takagi, T. Honjo and M. Ozawa: J. Japan Inst. Metals 59 (1995) 408-414, in Japanese.

23) M. Uda and S. Ohno: Nippon Kagaku Kaishi 1984 (1984) 862, in Japanese.

24) W. Mahoney, M. D. Kempe and R. P. Andres: Mat. Res. Soc. Symp. Proc. 400 (1996) 65-70. 